\title{
Improved differentiation between primary lung cancer and pulmonary metastasis by combining dual-energy CT-derived biomarkers with conventional CT attenuation
}

\author{
Dominik Deniffel $^{1,2} \cdot$ Andreas Sauter $^{1}$ (D) $\cdot$ Alexander Fingerle $^{1} \cdot$ Ernst J. Rummeny ${ }^{1} \cdot$ Marcus R. Makowski $^{1}$. \\ Daniela Pfeiffer ${ }^{1}$
}

Received: 24 April 2020 / Revised: 26 June 2020 / Accepted: 13 August 2020 / Published online: 28 August 2020

(C) The Author(s) 2020

\begin{abstract}
Objectives To assess the clinical utility of dual-energy CT (DE-CT)-derived iodine concentration (IC) and effective $Z$ ( $Z^{\text {eff }}$ ) in addition to conventional CT attenuation (HU) for the discrimination between primary lung cancer (LC) and pulmonary metastases (PM) from different primary malignancies.

Methods DE-CT scans of 79 patients with LC (3 histopathologic subgroups) and 89 patients with PM (5 histopathologic subgroups) were evaluated. Quantitative IC, $Z^{\text {eff }}$, and conventional HU values were extracted and normalized to the thoracic aorta. Differences between groups were assessed by pairwise Welch's $t$ test. Correlation and linear regression analyses were used to examine the relationship of imaging parameters in LC and PM. Diagnostic accuracy was measured by the area under receiver operator characteristic curve (AUC) and validated based on resampling methods.

Results Significant differences between subgroups of LC and PMs were noted for all imaging parameters, with the highest number of significant pairs for IC. In univariate analysis, only IC was a significant diagnostic feature for discriminating LC from PM $(p=0.03)$. All quantitative imaging parameters correlated significantly $\left(p<0.0001\right.$, respectively), with the highest correlation between IC and $Z^{\text {eff }}$ $(r=0.91)$, followed by IC and HU $(r=0.76)$ and $Z^{\text {eff }}$ and HU $(r=0.73)$. Diagnostic models combining IC or $Z^{\text {eff }}$ with HU (IC+HU: $\left.\mathrm{AUC}=0.73 ; \mathrm{Z}^{\mathrm{eff}}+\mathrm{HU}: \mathrm{AUC}=0.69 ; \mathrm{IC}+Z^{\text {eff }}+\mathrm{HU}: \mathrm{AUC}=0.73\right)$ were not significantly different and outperformed individual parameters ( $\left.\mathrm{IC}: \mathrm{AUC}=0.57 ; \mathrm{Z}^{\mathrm{eff}}: \mathrm{AUC}=0.57 ; \mathrm{HU}: \mathrm{AUC}=0.55\right)$ in diagnostic accuracy $(p<0.05$, respectively).

Conclusion DE-CT-derived IC or $Z^{\text {eff }}$ and conventional HU represent complementary imaging parameters, which, if used in combination, may improve the differentiation between LC and PM.

Key Points

- Individual quantitative imaging parameters derived from DE-CT (iodine concentration, effective Z) and conventional CT (HU) provide complementary diagnostic information for the differentiation of primary lung cancer and pulmonary metastases.

- A combination of conventional HU and DE-CT parameters enhances the diagnostic utility of individual parameters.
\end{abstract}

Keywords Neoplasms lung · Tomography X-ray computed $\cdot$ Lung cancer $\cdot$ Contrast media

Dominik Deniffel and Andreas Sauter contributed equally to this work.

Electronic supplementary material The online version of this article (https://doi.org/10.1007/s00330-020-07195-9) contains supplementary material, which is available to authorized users.

Andreas Sauter

andreas.sauter@tum.de

1 Department of Radiology, Klinikum rechts der Isar, School of Medicine, Technical University of Munich, Ismaninger Str. 22, 81675 Munich, Germany

2 Lunenfeld-Tanenbaum Research Institute, Sinai Health System, University of Toronto, Toronto, ON, Canada

$\begin{array}{ll}\text { Abbreviations } \\ \text { AUC } & \begin{array}{l}\text { Area under the receiver operating characteristic } \\ \text { curve }\end{array} \\ \text { CRC } & \text { Colorectal adenocarcinoma } \\ \text { CT } & \text { Computed tomography } \\ \text { CTDI }_{\text {vol }} & \text { Volume-based CT dose index } \\ \text { DE-CT } & \text { Dual-energy CT } \\ \text { DL-CT } & \text { Dual-layer CT } \\ \text { DLP } & \text { Dose length product } \\ \text { DS-CT } & \text { Dual-source CT } \\ \text { HU } & \text { Hounsfield units } \\ \text { IC } & \text { Iodine concentration }\end{array}$




$\begin{array}{ll}\text { LC } & \text { Lung cancer } \\ \text { PBC } & \text { Pancreato-biliary adenocarcinoma } \\ \text { PM } & \text { Pulmonary metastasis } \\ \text { RCC } & \text { Renal cell carcinoma } \\ \text { ROC } & \text { Receiver operating characteristic } \\ \text { ROI } & \text { Region-of-interest } \\ \text { SCC } & \text { Squamous cell carcinoma } \\ \text { SCLC } & \text { Small-cell lung cancer } \\ \text { SD } & \text { Standard deviation } \\ \text { SEM } & \text { Standard error of the mean }\end{array}$

\section{Introduction}

Computed tomography $(\mathrm{CT})$ represents the cornerstone modality for the imaging of pulmonary malignancies. Lung cancer (LC) is the most common cancer in the USA and causes the most cancer-related deaths per organ site [1]. However, the most common malignant tumor found in the lungs are pulmonary metastases (PM) originating from another primary cancer [2]. The differentiation between primary LC and PM is criti$\mathrm{cal}$, as it defines the diagnostic and therapeutic workflow. For example, diagnosis of PM usually warrants further imaging studies to identify the primary malignancy (e.g., mammography and other organ-specific imaging studies), whereas LC requires complete staging examinations before definitive treatment can be initiated. Additionally, patients may present with synchronous PM and primary LC [3] or with a known primary tumor and a new pulmonary lesion of unknown origin
(PM or LC). Until recently, upon appearance of an unknown pulmonary lesion on $\mathrm{CT}$, radiologists used morphological criteria, such as density, borders, and size, to narrow down the list of possible differential diagnoses $[4,5]$. These criteria are particularly useful for the differentiation between malignant and benign pulmonary lesions. However, in most cases, further subclassification of lesions is not possible based on conventional $\mathrm{CT}$ alone, ultimately requiring biopsy or follow-up examinations for definite diagnosis.

Over the last years, dual-energy CT (DE-CT) has emerged as a promising diagnostic technology for various clinical applications [6-10] leading to widespread adoption in clinical practice. Previous studies exploring the use of DE-CT for the assessment of pulmonary lesions focused on the differentiation between lung cancer (LC) and inflammatory masses [11] on the one hand and the differentiation of PMs $[12,13]$ on the other hand. To our knowledge, no previous studies have investigated the differentiation of LC and PM by conventional CT or DE$\mathrm{CT}$, despite the given clinical relevance. In previous DE-CT studies, the most frequently used imaging parameter was iodine concentration (IC). More recently, the effective atomic number $\left(Z^{\text {eff }}\right)$ decomposition of tissues was introduced for oncologic applications. Similar to IC, the rationale of using $Z^{\text {eff }}$ is accurate quantification of iodine uptake, considered a surrogate measure for tumor perfusion. Both imaging parameters are now readily available in commercial DE-CT analysis packages. This, however, has raised a further dilemma for radiologists in clinical practice: which quantitative imaging parameters should be used for the characterization of pulmonary lesions or can they be

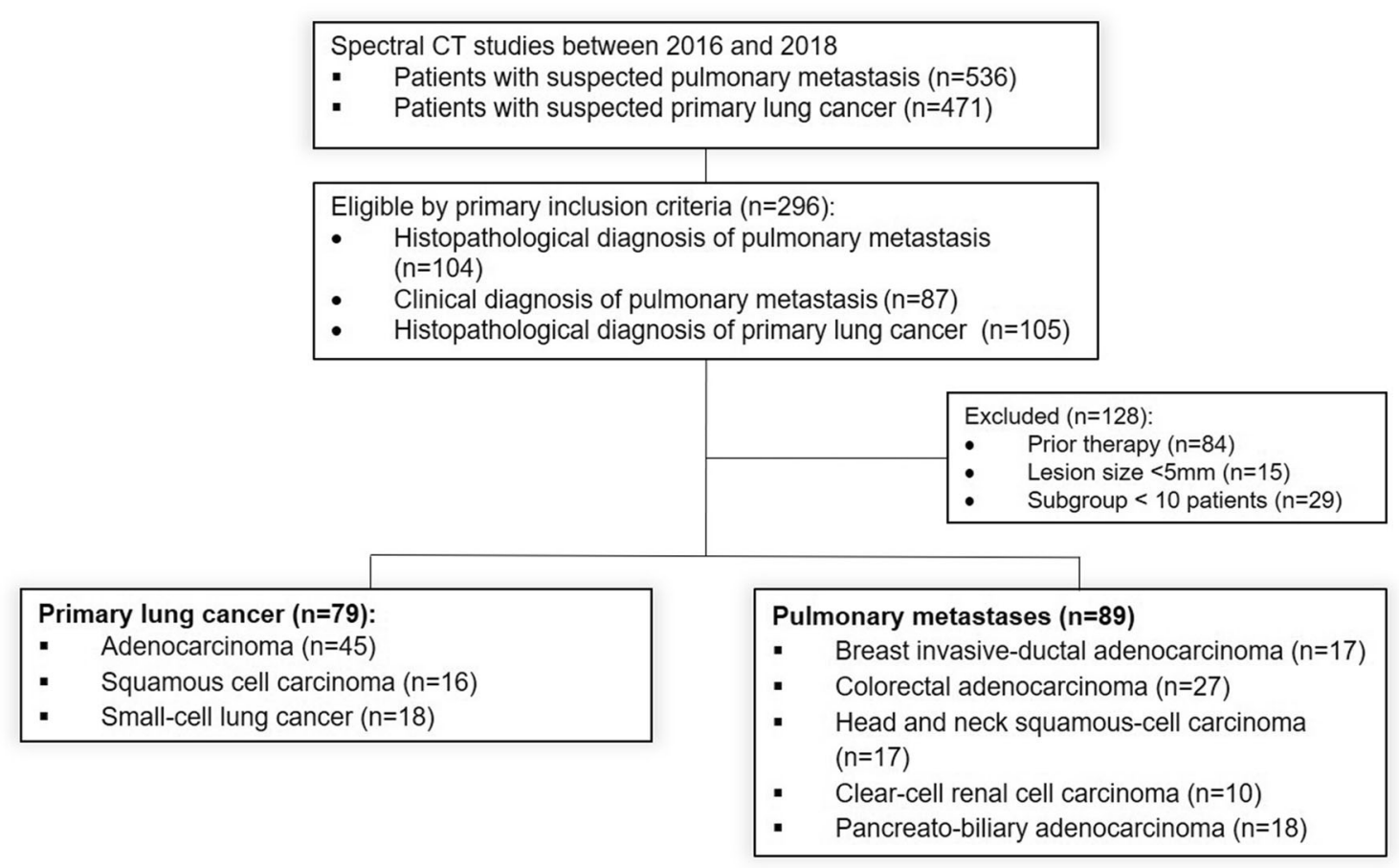

Fig. 1 Patient selection process 
Table 1 Iodine concentration, effective Z, conventional HU, and tumor volume of lung metastases and primary lung cancers

\begin{tabular}{|c|c|c|c|c|c|c|}
\hline Type & Primary location & Histology & $n$ & $\mathrm{IC}(\mathrm{mg} / \mathrm{ml})$ & $Z^{\text {eff }}$ & $\mathrm{HU}$ \\
\hline Metastasis & Breast & Invasive-ductal adenocarcioma & 17 & $1.5 \pm 0.59$ & $8.00 \pm 0.37$ & $60.39 \pm 18.95$ \\
\hline Metastasis & Colorectum & Adenocarcioma & 27 & $1.26 \pm 0.56$ & $7.97 \pm 0.38$ & $50.5 \pm 22.34$ \\
\hline Metastasis & Head and neck & Squamos cell carcinoma & 17 & $1.57 \pm 0.46$ & $8.21 \pm 0.27$ & $59.72 \pm 15.89$ \\
\hline Metastasis & Kidney & Clear-cell carcinoma & 10 & $2.89 \pm 1.24$ & $8.81 \pm 0.51$ & $94.39 \pm 22.77$ \\
\hline Metastasis & Pancreato-biliary tract & Adenocarcioma & 18 & $2.2 \pm 0.76$ & $8.43 \pm 0.35$ & $67.73 \pm 25.62$ \\
\hline Primary tumor & Lung & Adenocarcioma & 45 & $1.66 \pm 0.47$ & $8.18 \pm 0.21$ & $71.10 \pm 17.1$ \\
\hline Primary tumor & Lung & Squamos cell carcinoma & 16 & $1.44 \pm 0.78$ & $8.04 \pm 0.46$ & $65.98 \pm 20.78$ \\
\hline Primary tumor & Lung & Small-cell lung cancer & 18 & $1.09 \pm 0.42$ & $7.96 \pm 0.39$ & $55.53 \pm 14.2$ \\
\hline
\end{tabular}

Listed are mean values \pm standard deviation. Pulmonary tumors are specified by their type (primary, metastatic), location of their primary tumor, and histology. The following parameters were measured using dual-energy CT: $I C$, iodine concentration; $Z^{\text {eff }}$, effective atomic number; $H U$, conventional attenuation values in Hounsfield units

used interchangeably? Furthermore, would conventional Hounsfield units (HU) still provide an incremental diagnostic value or is this parameter obsolete when IC or $Z^{\text {eff }}$ measurements are available? Only few studies assessed all of the latter imaging parameters simultaneously and for pulmonary malignancies, no formal analysis has been presented as to which quantitative imaging parameters is to be preferred.

In this study, we sought to systematically assess the diagnostic value of IC and $Z^{\text {eff }}$, derived from DL-CT, as well as conventional HU for the characterization of numerous pulmonary malignancies. We hypothesized that incorporating IC, $Z^{\text {eff }}$, and HU into a diagnostic model may improve the differentiation between pulmonary metastases and LC and thus help streamline the diagnostic workflow in patients with pulmonary lesions of unknown origin.

\section{Material and methods}

\section{Patient population}

This retrospective, HIPAA-compliant, single-center study was approved by our institutional review board, and a waiver of informed consent was obtained. Between 2016 and 2018, 1007 patients underwent a DL-CT of the thorax for suspected
PM or suspected primary lung tumors. Primary inclusion criteria were (a) histopathologically confirmed primary LC, (b) histopathologically confirmed PM, and (c) clinically confirmed PM. Clinical diagnosis of PM was based on a followup $>6$ months demonstrating progression of the PM, defined by the appearance of new lesions or $>20 \%$ progression in size. Histopathological subgroups smaller than 10 patients were excluded from further analysis. Furthermore, patients were excluded due to prior therapy (local, systemic) and lesion size less than $5 \mathrm{~mm}$. We finally analyzed 79 patients with primary LC (adenocarcinoma, $n=45$; squamous cell carcinoma (SCC), $n=16$; small-cell LC (SCLC), $n=18$ ) and 89 patients with $\mathrm{PM}$ from primary breast (invasive-ductal adenocarcinoma, $n=17$ ), colorectal (CRC) (adenocarcinoma, $n=$ 27), head and neck (squamous cell carcinoma, $n=17$ ), kidney (RCC) (clear-cell renal cell carcinoma, $n=10$ ), and pancreatobiliary (PBC) (adenocarcinoma, $n=18$ ) malignancies. The study population flowchart is illustrated in Fig. 1.

\section{Dual-energy CT technique}

The examination of all patients using a dual-layer spectral CT (IQon; Philips Healthcare) followed the same routine protocol. Before the start of the scan, an anterior-posterior scout was performed to determine the scan range. Intravenous
Fig. 2 Examples of a patient with primary lung cancer (left) and with pulmonary metastasis (right). In each patient, a lesion in the apex of the left upper lobe is present. The following values were measured for these lesions: HU: 61/68; $Z^{\text {eff }: ~ 8.4 / 7.3 ; ~ I C ~ 1.84 / ~}$ 0.60

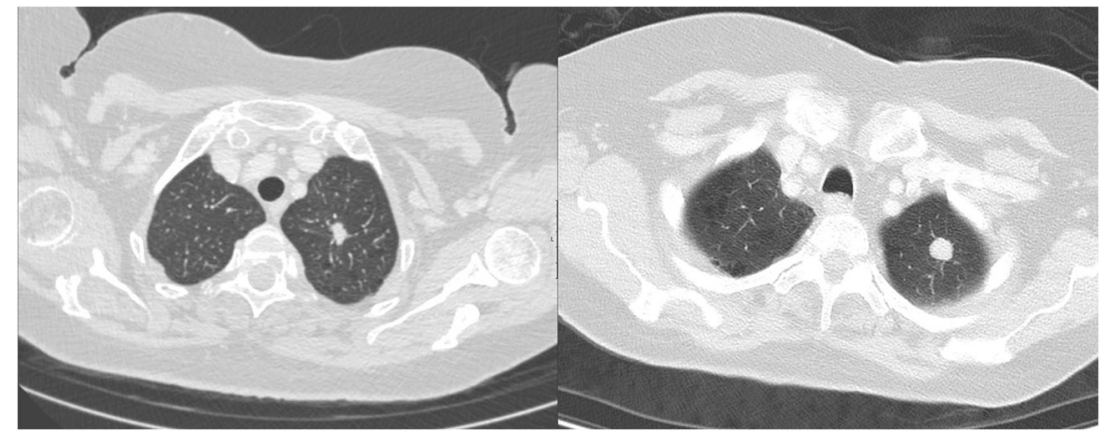



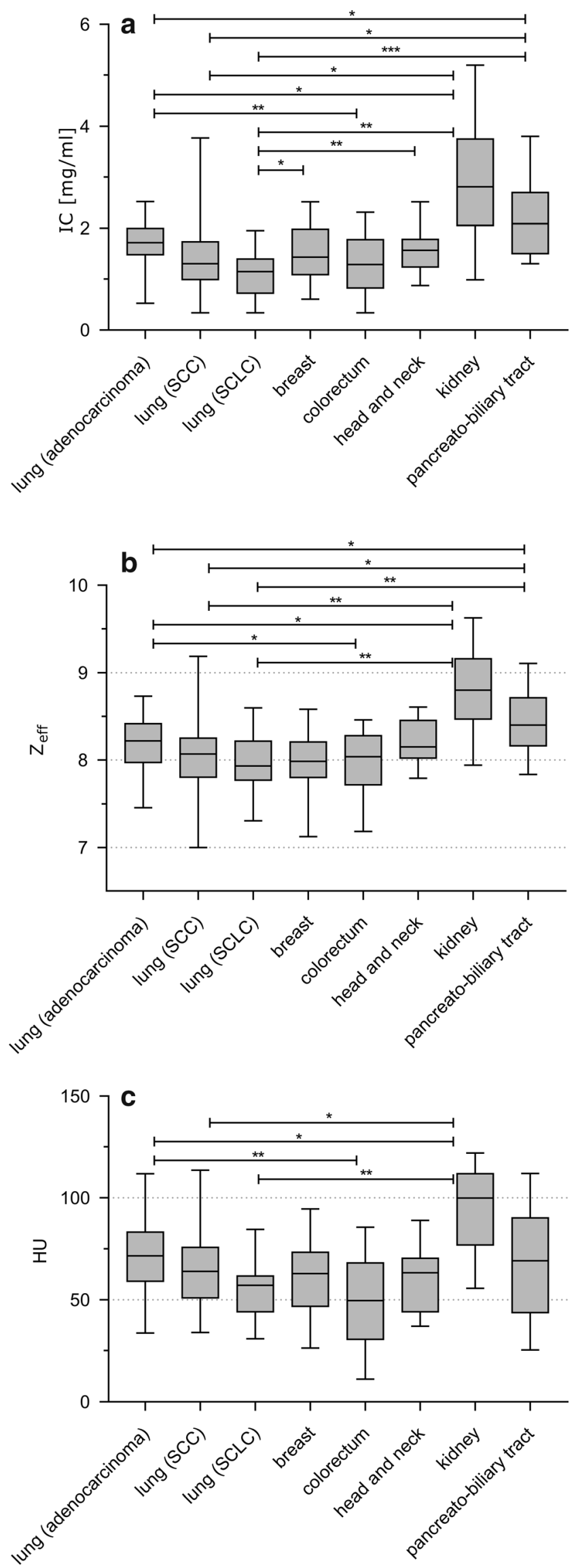

Fig. 3 Quantitative results for the dual-energy CT-derived iodine concentration and conventional $\mathrm{CT}$ attenuation values. a Median values of iodine concentration (IC), (b) effective atomic number ( $\mathrm{Z}^{\mathrm{eff}}$ ), and (c) conventional Hounsfield unit $(\mathrm{HU})$ values with quartiles, minimum, and maximum values, for primary lung cancers (adenocarcinoma, squamous cell carcinoma (SCC), small-cell lung cancer (SCLC)) and pulmonary metastases from primary breast (invasive-ductal adenocarcinoma), colorectal (adenocarcinoma), head and neck (squamous cell carcinoma), kidney (clear-cell renal cell carcinoma), and pancreato-biliary (adenocarcinoma) malignancies. Presented are $p$ values of pairwise Welch $t$ tests adjusted for multiple comparisons using the BenjaminiHochberg correction. Differences between subgroups of pulmonary metastases and primary lung cancers were not assessed. *Adjusted $p$ value of $<0.05, * *<0.01, * * *<0.001$

contrast medium (Imeron $400 \mathrm{MCT}, 400 \mathrm{mg} / \mathrm{ml}$; Bracco Imaging) was injected with a standard dosage of $80 \mathrm{ml}$ at a flow rate of $2-2.5 \mathrm{ml} / \mathrm{s}$, followed by a $30-\mathrm{ml}$ saline chaser at the same flow rate. All scans were performed in the venous phase (scan delay $70 \mathrm{~s}$ after the start of the application of the contrast medium). The following scanning parameters were used: $120 \mathrm{kVp}$; automatic tube current selection with resulting exposures of 37-84 mAs; $512 \times 512$ matrix; collimation $64 \times$ $0.625 \mathrm{~mm}$; reconstructed slice thickness and interval $0.9 \mathrm{~mm} /$ $0.9 \mathrm{~mm}$ with a soft tissue kernel. The field-of-view was adapted to the patient size. Conventional and spectral basis images were reconstructed using the iDose ${ }^{4}$ (Philips Healthcare) algorithm. The mean volume-weighted CT dose index $\left(\mathrm{CTDI}_{\mathrm{vol}}\right)$ and dose length product (DLP) for the complete protocol were $4.4 \mathrm{mGy}$ and $180 \mathrm{mGy} * \mathrm{~cm}$, respectively, corresponding to an effective dose of $2.5 \mathrm{mSv}$ (conversion factor 0.014).

\section{Image analysis}

Quantitative analysis of spectral CT images was performed using commercially available software (IntelliSpace Portal v. 10.1, Philips Healthcare). Image analysis was performed retrospectively by a resident fellow in radiology and supervised by a senior radiologist (11 years of radiology experience). Using a semi-automated 3D segmentation algorithm, a volume-of-interest was created for each pulmonary lesion. A circular two-dimensional region-of-interest was manually placed in the descending aorta. The following imaging parameters were acquired: conventional (polychromatic) Hounsfield units (HU), iodine concentration (IC) $(\mathrm{mg} / \mathrm{ml})$, and effective atomic number $\left(Z^{\text {eff }}\right)$. To account for hemodynamic interpatient variations, the measured parameter values in the volume-of-interest of the PM were normalized to the thoracic aorta as previously described [13]. All analyses were conducted on normalized measurements.

\section{Statistical analysis}

Reporting followed Standards of Reporting of Diagnostic Accuracy [14]. All statistical analyses were performed using 
Table 2 Linear regression analysis

\begin{tabular}{llllc}
\hline Model & Dependent variable & Independent variables & Coeffecient $[\mathrm{CI}]$ & $p$ value \\
\hline a & HU & IC & $23.36[19.64,26.49]$ & $<0.0001$ \\
& & Primary lung cancer & $9.85[5.4,13.85]$ & $<0.0001$ \\
& & Pulmonary metastasis & Reference & \\
b & HU & Z & $39.9[34.12,44.72]$ & $<0.0001$ \\
& & Primary lung cancer & $8.43[3.63,12.62]$ & 0.0003 \\
& & Pulmonary metastasis & Reference & \\
c & IC & Primary lung cancer & $0.50[0.46,0.55]$ & $<0.0001$ \\
& & Pulmonary metastasis & Reference & 0.59 \\
& & &
\end{tabular}

CI indicates bootstrapped 95\% confidence interval (2000 replicates). IC, iodine concentration on dual-energy computed tomography; $H U$, conventional computed tomography attenuation values in Hounsfield units; $Z^{\text {eff }}$, effective atomic number. Italics indicate significant $p$ values $(<0.05)$. Note that two-way interaction terms for all three models were not significant in preliminary analyses
$\mathrm{R}$ version 3.6.1 (R Foundation for Statistical Computing) and the following $\mathrm{R}$ packages: caret (versions 6.0-84), boot (versions 1.3-22), car (versions 3.0-3), pROC (1.15.3) [15]. Confidence intervals for all analyses were derived using bootstrap sampling with 2000 replicates. Differences between means were assessed by pairwise Welch's $t$ test to correct for unequal variances between groups. Multiple testing adjusted $p$ values were computed using Benjamini-Hochberg correction [16], also known as false-discovery rate. Differences were only assessed between subgroups of metastases and LC. The relationship between imaging parameters was evaluated using the Pearson correlation coefficient $(r)$ and linear regression analysis. Threeparameter linear regression models were constructed using pairs of imaging parameters as independent and dependent variable, the type of pulmonary tumor as categorical variable (primary versus metastatic), and two-way interactions between them. This approach allows to assess the change of one imaging parameter value in a tumor subgroup as a function of another imaging parameter. Logistic regression models were developed for the discrimination of primary LC versus PM using imaging parameters alone and in combination. Bootstrapping with 2000 replications was performed for internal validation to avoid optimism in model performance measures. Diagnostic accuracy was measured by the area under receiver operator characteristic (ROC) curve (AUC) and compared using a bootstrap test (2000 replicates) [15]. Additional diagnostic performance measures (sensitivity, specificity, and negative and positive predictive value) were calculated for model thresholds corresponding to the maximum Youden index. All tests were two-tailed, and $p<0.05$ was considered statistically significant.

\section{Results}

A total of 79 LC and 89 PM on 168 DE-CT scans were analyzed in this study; a representative example of a LC and a PM is shown in Fig. 2. Quantitative results of $\mathrm{IC}, \mathrm{Z}^{\mathrm{eff}}$, and conventional $\mathrm{CT}$ values of all pulmonary tumors are provided in Table 1 and Fig. 3a-c. Significant differences in all imaging parameters were observed for lung adenocarcinoma versus PM from CRC (IC: $p=0.01 ; Z^{\text {eff. }} p=0.05$; HU: $p=0.002$ ) and RCC (IC: $p=0.02 ; Z^{\text {eff. }} p=0.01 ; \mathrm{HU}: p=0.04$ ), for SCC versus PM from RCC (IC: $p=0.02 ; Z^{\text {eff }}: p=0.005$; HU: $p=$ 0.02 ), and for SCLC versus PM from RCC (IC: $p=0.009$; $\mathrm{Z}^{\text {eff. }} p=0.003$; HU: $\left.p=0.002\right)$. Additional significant differences were noted based on IC and $Z^{\text {eff }}$ between lung adenocarcinoma (IC: $\left.p=0.02 ; Z^{\text {eff: }} p=0.02\right)$, SCC (IC: $p=0.02$; $\mathrm{Z}^{\text {eff. }} p=0.02$ ), and SCLC (IC: $p=0.0001 ; Z^{\text {eff }}: p=0.003$ ) versus metastatic lesions from $\mathrm{PBC}$, respectively. Significant differences between SCLC and metastases from head and neck cancer (IC: $p=0.01$ ) and breast cancer (IC: $p=0.04$ ) were only observed for IC.

All quantitative imaging parameters correlated significantly, with the highest correlation between IC and $\mathrm{Z}^{\text {eff }}(r=0.91, p$ $<0.0001)$, followed by IC and HU $(r=0.76, p<0.0001)$ and $\mathrm{Z}^{\text {eff }}$ and HU $(r=0.73, p<0.0001)$. We analyzed the following linear regression models including the type of pulmonary tumor (primary versus metastatic) and pairs of imaging parameter as dependent versus independent variable (Table 2): model (a) HU versus IC (Fig. 4a); model (b) HU versus $Z^{\text {eff }}$ (Fig. 4b); model (c) $Z^{\text {eff }}$ versus IC (Fig. 4c). A two-way interaction term was not statistically significant in any of the three regression models (model a: $p=0.94$; model b: $p=0.53$; model c: $p=0.13$ ) and was, therefore, dropped from the models. A significant effect of the type of pulmonary tumor on HU values (dependent variable) was observed for models a $(p<0.0001)$ and $\mathrm{b}(p<0.0001)$, with coefficients of 9.85 and 8.43 , respectively, for the category "Primary LC. These results translate into significantly higher HU values of LCs compared with those of PM at fixed values of IC (model a) and $\mathrm{Z}^{\text {eff }}$ (model b), and, by contrast, no significant differences in $Z^{\text {eff }}$ at fixed values of IC (model c). 

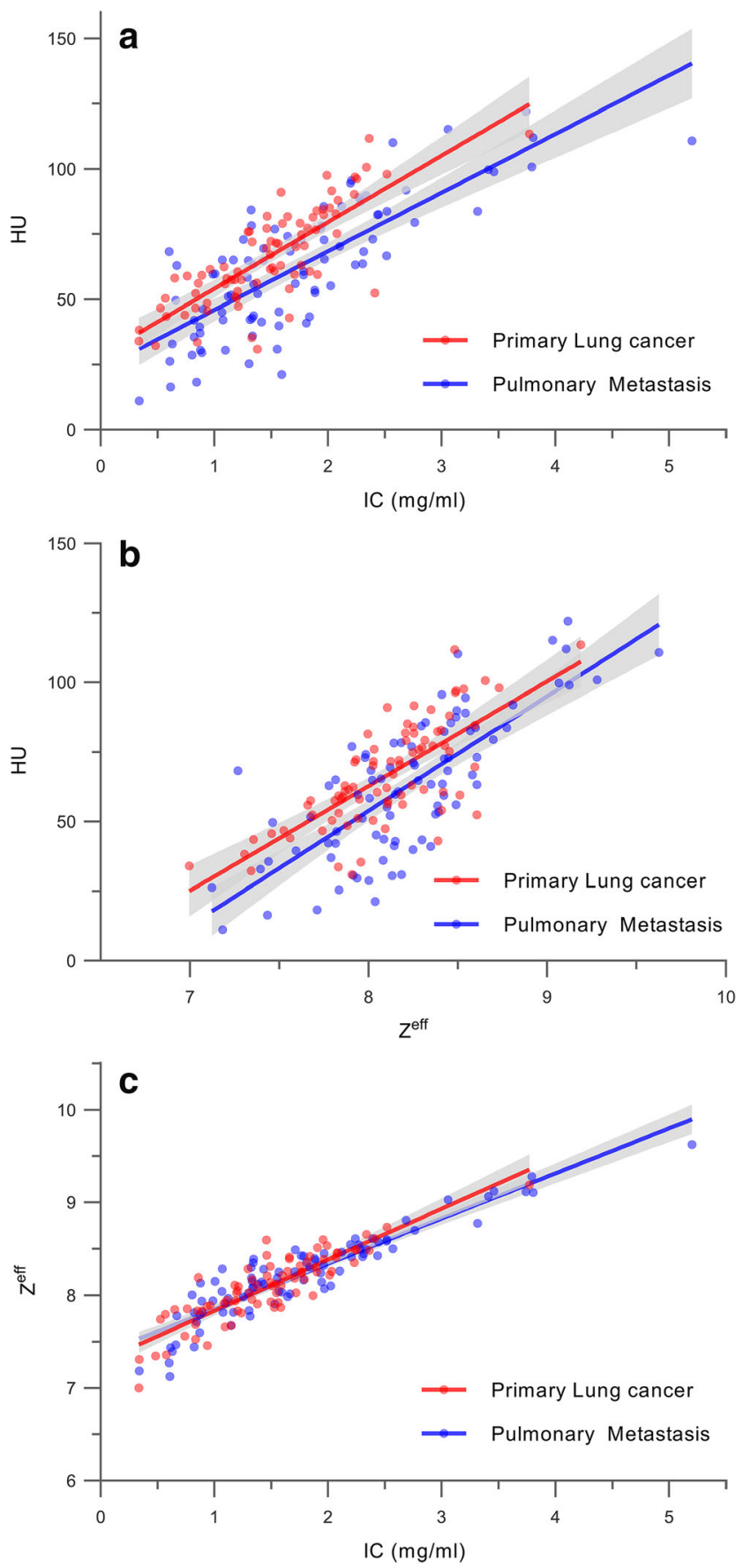

Fig. 4 Linear regression scatter plots of quantitative imaging parameters in primary lung cancer and pulmonary metastases. a Conventional HU and IC. b Conventional HU and $Z^{\text {eff }}$. $\mathbf{~ Z ~} Z^{\text {eff }}$ and IC. Gray-shaded area represents the limits of the $95 \%$ confidence interval of the linear regression fit. Significant differences in HUs were noted between primary lung cancer and pulmonary metastases at fixed IC $(p<0.0001)$ and $Z^{\text {eff }}(p=0.0003)$ in regression models $\mathbf{a}$ and $\mathbf{b}$; no significant difference in $Z^{\text {eff }}$ at fixed IC was found in model $\mathbf{c}$

In univariate logistic regression analysis, only IC was found to be a significant predictor for the discrimination of primary LC from PM ( $p=0.03$; Table 3$)$. Coefficients of uni- and multivariate logistic regression models are
Table 3 Univariate logistic regression analysis for discrimination of primary lung cancer from pulmonary metastases

\begin{tabular}{llll}
\hline Variable & Coeffecient & {$[\mathrm{CI}]$} & $p$ value \\
\hline IC & -0.481 & {$[-0.95,-0.07]$} & 0.03 \\
$\mathrm{Z}^{\text {eff }}$ & -0.66 & {$[-1.36,0.13]$} & 0.09 \\
$\mathrm{HU}$ & 0.01 & {$[-0.01,0.02]$} & 0.25 \\
\hline
\end{tabular}

CI indicates bootstrapped $95 \%$ confidence intervals (2000 replicates). IC, iodine concentration on dual-energy computed tomography; $H U$, conventional computed tomography attenuation values in Hounsfield units; $Z^{\text {eff }}$, effective atomic number. Italic indicates significant $p$ values $(<0.05)$

provided in Supplementary Table 1. Diagnostic performance was assessed for all imaging parameters alone and in combination (Table 4; Supplementary Table 2). Only the corresponding AUCs of logistic regression models combining $\mathrm{HU}$ with IC and/or $\mathrm{Z}^{\text {eff }}$ were significantly different from 0.5 ( $p<0.0001$, respectively), indicating useful diagnostic tests. Accordingly, the corresponding bootstrap optimism-corrected AUCs of these combinations were significantly higher compared with those of single imaging parameters and a combination of IC and $Z^{\text {eff }}(p<0.005$, respectively). The combined models including $\mathrm{HU}\left(\mathrm{IC}+\mathrm{HU}, \mathrm{Z}^{\text {eff }}+\mathrm{HU}, \mathrm{IC}+\mathrm{Z}^{\text {eff }}+\mathrm{HU}\right)$ did not differ significantly in their corresponding AUCs. The highest AUC was achieved by the parameter combination of IC and HU (AUC 0.73) (Table 4; Fig. 5).

\section{Discussion}

Our study demonstrates that the discrimination of primary LC from PM is feasible using a combination of DE-CT-derived IC or $Z^{\text {eff }}$, and conventional HU. Using either imaging parameter alone did not enable diagnostic utility. IC and $Z^{\text {eff }}$ did not provide complementary information to one another for this diagnostic task and can thus be used interchangeably for the implementation into a diagnostic model.

In earlier reports, DE-CT-derived parameters were used for the differentiation of PM from different primary tumors [12, 13] and for the evaluation of therapy response in LC [17]. However, to our knowledge, no previous studies have examined the differentiation between LC and PM. For the majority of cases in current clinical practice, this discrimination can only be made based on histopathological evaluation. Therefore, patients are required to undergo biopsy upon appearance of new pulmonary lesions on CT. Aside from possible complications, such as pneumothorax or bleeding, this diagnostic pathway may ultimately delay further therapy. Our study suggests DE-CT as a useful tool for the differentiation of LC and PM, which may help streamline the diagnostic workflow in patients with pulmonary lesions of unknown 
Table 4 Diagnostic accuracy for the differentiation of primary lung cancer from pulmonary metastases

\begin{tabular}{lllllllll}
\hline Model & AUC [CI] & $p^{\mathrm{a}}$ & $p^{\mathrm{b}}$ & $p^{\mathrm{c}}$ & $p^{\mathrm{d}}$ & $p^{\mathrm{e}}$ & $p^{\mathrm{f}}$ & $p^{\mathrm{g}}$ \\
\hline $\mathrm{IC}$ & $0.57[0.48,0.65]$ & & 0.95 & 0.83 & 0.0009 & 0.02 & 1 & 0.001 \\
$\mathrm{Z}^{\text {eff }}$ & $0.57[0.48,0.66]$ & 0.95 & & 0.82 & 0.002 & 0.01 & 0.96 & 0.002 \\
$\mathrm{HU}$ & $0.55[0.46,0.63]$ & 0.83 & 0.82 & & 0.0003 & 0.006 & 0.83 & 0.0003 \\
$\mathrm{IC}+\mathrm{HU}$ & $0.73^{*}[0.65,0.80]$ & 0.0009 & 0.002 & 0.0003 & & 0.17 & 0.0009 & 0.36 \\
$\mathrm{Z}^{\text {eff }}+\mathrm{HU}$ & $0.69^{*}[0.61,0.77]$ & 0.02 & 0.01 & 0.006 & 0.17 & & 0.04 & 0.17 \\
$\mathrm{IC}+Z^{\text {eff }}$ & $0.57[0.48,0.65]$ & 1 & 0.96 & 0.83 & 0.0009 & 0.04 & & 0.0009 \\
$\mathrm{IC}+Z^{\text {eff }}+$ & $0.73^{*}[0.65,0.80]$ & 0.001 & 0.002 & 0.0003 & 0.36 & 0.17 & 0.0009 & \\
$\quad \mathrm{HU}$ & & & & & & & & \\
\hline
\end{tabular}

$A U C$, area under the receiver operating characteristic curve; $C I$, bootstrapped $95 \%$ confidence intervals (2000 replicates); $I C$, iodine concentration on dual-energy computed tomography; $H U$, conventional computed tomography attenuation values in Hounsfield units; $Z^{\text {eff }}$, effective atomic number. $p$ values are provided for the comparisons of bootstrap optimism-corrected AUCs: versus ${ }^{\mathrm{a}} \mathrm{IC} ;{ }^{\mathrm{b}} \mathrm{Z}^{\mathrm{eff}} ;{ }^{\mathrm{c}} \mathrm{HU} ;{ }^{\mathrm{d}} \mathrm{IC}+\mathrm{HU} ;{ }^{\mathrm{e}} \mathrm{Z}^{\mathrm{eff}}+\mathrm{HU} ;{ }^{\mathrm{f}} \mathrm{IC}+\mathrm{Z}^{\text {eff }} ;{ }^{\mathrm{g}} \mathrm{IC}+$ $Z^{\text {eff }}+$ HU. Italicized $p$ values indicate significant differences $(<0.05)$. *AUCs significantly different from 0.5 origin and even avoid invasive diagnostic procedures in certain cases.

Our study focused on the utility of the most commonly used DE-CT-derived parameters IC and $Z^{\text {eff }}$, which are readily provided by most commercial software platforms, in addition to conventional HU. While previous DE-CT studies used a multitude of imaging features, such as the slope of the spectral HU curve [18], virtual mono-energetic HUs, or extracted radiomic features from $\mathrm{DE}-\mathrm{CT}$-derived parametric maps
[19], the appeal of our approach is its simplicity, only using three "off-the-shelf imaging parameters obtained from DECT scans which could facilitate integration into the clinical workflow without additional software applications.

Our study could demonstrate that a differentiation between LC and PM is possible in many cases. However, if used alone, no parameter provided reliable predictions. Both $\mathrm{IC}$ and $\mathrm{Z}^{\text {eff }}$ are considered surrogate measures for tumor vascularity and perfusion. While IC directly quantifies iodine content, $Z^{\text {eff }}$
Fig. 5 ROC curves for the discrimination of primary lung cancer $(n=79)$ from pulmonary metastases $(n=89)$ corrected for optimism using bootstrapping (2000 replicates). Logistic regression models, combining dual-energy CT-derived iodine concentration (IC), $Z^{\text {eff }}$, and conventional $\mathrm{CT}$ attenuation values (HU), as well as single parameters, were tested. The corresponding areas under the curve (AUCs) of the combined models incorporating $\mathrm{HU}$ were significantly higher than those of IC $(p<0.05), Z^{\text {eff }}(p<0.01)$, or $\mathrm{HU}(p<0.01)$ alone. No significant differences were noted between single parameters and a combined model using IC and $Z^{\text {eff }}$

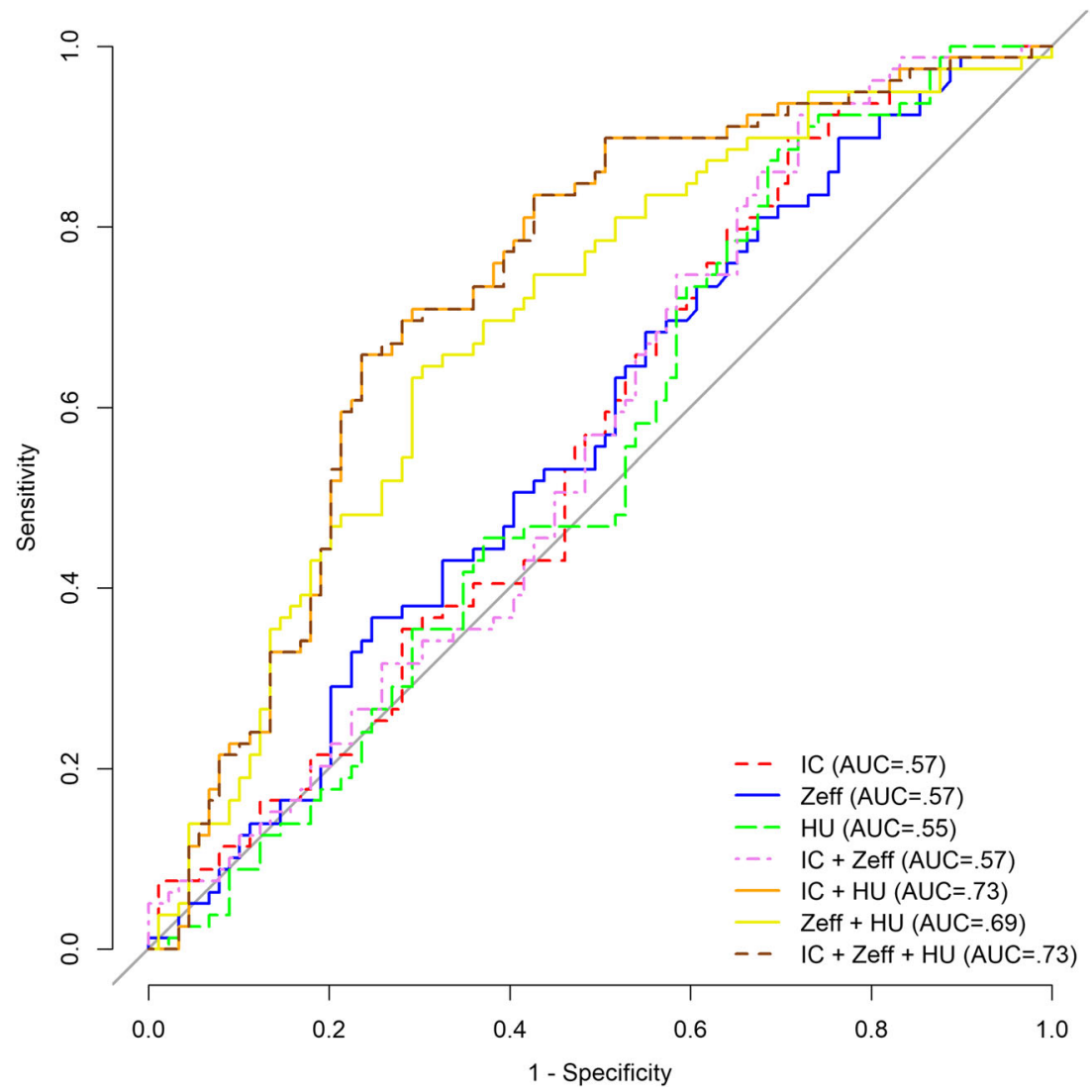


describes the average atomic number for a tissue of interest and can thus indirectly provide information about accumulation of contrast material. Coefficients for IC and $\mathrm{Z}^{\text {eff }}$ in the logistic regression models indicate that $\mathrm{LC}$ accumulated less contrast material than PMs, indicating differences in tumor vascularity. Nonetheless, diagnostic utility could only be achieved by the addition of HU in a diagnostic model. This diagnostic incremental value of HU was confirmed by linear regression analysis. At fixed values of IC or $\mathrm{Z}^{\text {eff }}$, LCs demonstrated significantly higher HU values than PMs. This suggests that, in addition to tumor vascularity, which may be accurately assessed by IC or $Z^{\text {eff }}$, there are subtle differences in tissue composition that are more evident by HU quantification. Consequently, the assessment of pulmonary tumors of unknown origin in clinical routine should not be limited to single parameters but consider both conventional CT attenuation and DE-CT-derived parameters.

Not all pairwise comparisons between subgroups of LC and PM in our study yielded significant differences for all imaging parameters. Given the similarity of several subgroups of LC and PM, in many cases, a definite differentiation may not be achieved by imaging alone. In a clinical context, our proposed combined diagnostic models could, however, provide an additional diagnostic hint and help streamline further diagnostic work-up. For instance, upon appearance of a new pulmonary lesion in patients with a known primary tumor, characteristic DE-CT parameter values may help to confirm the clinical suspicion and initiate therapy without additional invasive tissue sampling.

Findings of our study should be interpreted in the context of some limitations. First, the analysis was retrospective and limited to a single reference center. Second, the number of included CT scans for some histopathological subgroups was relatively low and, although we assessed a multitude of tumor types, no benign pulmonary lesions and not all malignant pulmonary tumors are considered. Third, no other DECT technology was used besides DL-CT as different systems are not available at our institution. Since IC can be measured accurately with other systems [20], the results of the present study are expected to be transferable to other systems. Fourth, we acknowledge that additional imaging characteristics such as tumor margins, size, calcifications, or presence of necrotic areas, which are an integral part of any clinical CT assessment, could have improved the diagnostic performance of our model. This study is a first step in demonstrating the feasibility of distinguishing between LC and PM. Machine learning algorithms handling multiple predefined input variables or deep learning models that are capable of automatically learning and extracting imaging features are an interesting avenue for future research but will ultimately require larger data sets.

In conclusion, our study demonstrates the feasibility of differentiating between LC and PM via DE-CT. Using a combination of IC and/or $Z^{\text {eff }}$ with HU may help avoid invasive tissue sampling in patients with a history of extra- or intrapulmonary malignancies.

Funding Open Access funding provided by Projekt DEAL. The study was supported by the German Research Foundation (DFG) within the Research Training Group GRK 2274. D.D. receives funding by the DFG (Fellowship DE 3207/1-1).

\section{Compliance with ethical standards}

Guarantor The scientific guarantor of this publication is Dr. Andreas P. Sauter.

Conflict of interest The authors of this manuscript declare no relationships with any companies whose products or services may be related to the subject matter of the article.

Statistics and biometry No complex statistical methods were necessary for this paper.

Informed consent Written informed consent was waived by the Institutional Review Board.

Ethical approval Institutional Review Board approval was obtained.

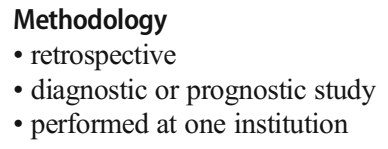

- performed at one institution

Open Access This article is licensed under a Creative Commons Attribution 4.0 International License, which permits use, sharing, adaptation, distribution and reproduction in any medium or format, as long as you give appropriate credit to the original author(s) and the source, provide a link to the Creative Commons licence, and indicate if changes were made. The images or other third party material in this article are included in the article's Creative Commons licence, unless indicated otherwise in a credit line to the material. If material is not included in the article's Creative Commons licence and your intended use is not permitted by statutory regulation or exceeds the permitted use, you will need to obtain permission directly from the copyright holder. To view a copy of this licence, visit http://creativecommons.org/licenses/by/4.0/.

\section{References}

1. Noone A, Howlader N, Krapcho M et al (2018) SEER cancer statistics review, 1975-2015. National Cancer Institute, Bethesda, MD

2. Rubin E, Reisner HM (2014) Essentials of Rubin's pathology. Wolters Kluwer Health/Lippincott Williams \& Wilkins, Philadelphia

3. Jin B, Zhang S, Chuang X et al (2018) Breast cancer and synchronous multiple primary lung adenocarcinomas with heterogeneous mutations: a case report. BMC Cancer 18:1138

4. Ruparel M, Quaife SL, Navani N, Wardle J, Janes SM, Baldwin DR (2016) Pulmonary nodules and CT screening: the past, present and future. Thorax 71:367-375

5. MacMahon H, Naidich DP, Goo JM et al (2017) Guidelines for management of incidental pulmonary nodules detected on CT images: from the Fleischner Society 2017. Radiology 284:228-243 
6. Sauter AP, Muenzel D, Dangelmaier J et al (2018) Dual-layer spectral computed tomography: virtual non-contrast in comparison to true non-contrast images. Eur J Radiol 104:108-114

7. Desai MA, Peterson JJ, Garner HW, Kransdorf MJ (2011) Clinical utility of dual-energy CT for evaluation of tophaceous gout. Radiographics 31:1365-1375 discussion 1376-1367

8. McCollough CH, Leng S, Yu L, Fletcher JG (2015) Dual- and multi-energy CT: principles, technical approaches, and clinical applications. Radiology 276:637-653

9. Lu GM, Wu SY, Yeh BM, Zhang LJ (2010) Dual-energy computed tomography in pulmonary embolism. Br J Radiol 83:707-718

10. Zhang LJ, Yang GF, Wu SY, Xu J, Lu GM, Schoepf UJ (2013) Dual-energy CT imaging of thoracic malignancies. Cancer Imaging 13:81-91

11. Hou WS, Wu HW, Yin Y, Cheng JJ, Zhang Q, Xu JR (2015) Differentiation of lung cancers from inflammatory masses with dual-energy spectral CT imaging. Acad Radiol 22:337-344

12. Altenbernd J, Wetter A, Umutlu L et al (2016) Dual-energy computed tomography for evaluation of pulmonary nodules with emphasis on metastatic lesions. Acta Radiol 57:437-443

13. Deniffel D, Sauter A, Dangelmaier J, Fingerle A, Rummeny EJ, Pfeiffer D (2019) Differentiating intrapulmonary metastases from different primary tumors via quantitative dual-energy CT based iodine concentration and conventional CT attenuation. Eur J Radiol 111:6-13

14. Bossuyt PM, Reitsma JB, Bruns DE et al (2003) Towards complete and accurate reporting of studies of diagnostic accuracy: the
STARD initiative. Standards for reporting of diagnostic accuracy. Clin Chem 49:1-6

15. Robin X, Turck N, Hainard A et al (2011) pROC: an open-source package for $\mathrm{R}$ and $\mathrm{S}+$ to analyze and compare ROC curves. BMC Bioinformatics 12:77

16. Benjamini Y, Hochberg Y (1995) Controlling the false discovery rate: a practical and powerful approach to multiple testing. J R Stat Soc B Methodol:289-300

17. Baxa J, Matouskova T, Krakorova G et al (2016) Dual-phase dualenergy $\mathrm{CT}$ in patients treated with erlotinib for advanced non-small cell lung cancer: possible benefits of iodine quantification in response assessment. Eur Radiol 26:2828-2836

18. Chen ML, Li XT, Wei YY, Qi LP, Sun YS (2019) Can spectral computed tomography imaging improve the differentiation between malignant and benign pulmonary lesions manifesting as solitary pure ground glass, mixed ground glass, and solid nodules? Thoracic Cancer 10:234-242

19. Choe J, Lee SM, Do KH et al (2019) Prognostic value of radiomic analysis of iodine overlay maps from dual-energy computed tomography in patients with resectable lung cancer. Eur Radiol 29:915923. https://doi.org/10.1007/s00330-018-5639-0

20. Sellerer T, Noel PB, Patino M et al (2018) Dual-energy CT: a phantom comparison of different platforms for abdominal imaging. Eur Radiol. https://doi.org/10.1007/s00330-017-5238-5

Publisher's note Springer Nature remains neutral with regard to jurisdictional claims in published maps and institutional affiliations. 